JOURNAL OF

SYMPLECTIC GEOMETRY

Volume 1 Number 4, 695-714, 2002

\title{
COSPHERE BUNDLE REDUCTION IN CONTACT GEOMETRY
}

\author{
Oana Drăgulete, Liviu Ornea, Tudor S. Ratiu
}

We extend the theorems concerning the equivariant symplectic reduction of the cotangent bundle to contact geometry. The role of the cotangent bundle is taken by the cosphere bundle. We use Albert's method for reduction at zero and Willett's method for non-zero reduction.

\section{Introduction}

One of the main results concerning symplectic reduction with many applications in geometric mechanics states that, in the presence of a "good" action of a finite dimensional Lie group $G$ on an arbitrary differentiable manifold $Q$, the cotangent bundle of the quotient, $T^{*}(Q / G)$, is symplectomorphic with $\left(T^{*} Q\right)_{0}$, the reduced space at 0 of the cotangent bundle. More generally, the reduction $T^{*}(Q / G)_{\mu}$ at $\mu \neq 0$ of $T^{*} Q$ is symplectomorphic with a vector subbundle of $T^{*}\left(Q / G_{\mu}\right)$ endowed with a magnetic symplectic form (see [1],$\S 4.3$; this result for Abelian groups is due to Satzer [10]); $G_{\mu}$ denotes the coadjoint isotropy subgroup at $\mu$.

The aim of this note is to prove an analogue of this result in contact geometry. Again we start with an arbitrary manifold $Q$ supporting a "good" action of a Lie group $G$. The role of the cotangent bundle will be played by the cosphere bundle that will be described in section 2 (cf. also [9]). It is a contact manifold. We shall prove that its reduced space at 0 is contactomorphic with the cosphere bundle of

Mathematics Subject Classification. 53D20, 53D10.

Key words and phrases. contact manifold, symplectic manifold, cotangent bundle, cosphere bundle, momentum map, (non-zero) reduction. 
$Q / G$. Even though the result for $\mu=0$ could probably be obtained by "diagram chasing", we prefer to provide an explicit proof, identifying all contactomorphisms. More generally, we prove that its reduced space at $\mu \neq 0$ embeds in a contact manner onto a subbundle of the cosphere bundle of $Q / G_{\mu}$.

We briefly review, following [2], [4], the reduction method at 0 for contact manifolds.

Recall that a contact structure on a smooth $(2 n+1)$-dimensional manifold $N$ is a codimension one smooth distribution $H \subset T N$, locally given by the kernel of a one-form $\eta$ such that $\eta \wedge(d \eta)^{n} \neq 0$. Such an $\eta$ is called a (local) contact form. Any two proportional contact forms underly the same contact structure. A contact structure which is the kernel of a global contact form is called exact or co-orientable. If $\eta$ is a one form of an exact contact structure, the pair $(N, \eta)$ is called an exact contact manifold. On an exact contact manifold $N$ there is a unique vector field $R$, called the Reeb vector field, characterized by the conditions $\eta(R)=1$ and $d \eta(R, \cdot)=0$. The flow of the Reeb vector fields preserves the contact form $\eta$. The Reeb vector field is nowhere vanishing and it generates the one-dimensional distribution ker $d \eta=\{v \in T N \mid d \eta(v, \cdot)=0\}$.

A finite dimensional connected Lie group $G$ is said to act by contactomorphisms on a contact manifold if it preserves the contact structure $H$. For an exact contact manifold $(N, \eta)$, this means that $g^{*} \eta=f_{g} \eta$ for a smooth, real-valued, nowhere zero function $f_{g}$. $G$ acts by strong contactomorphisms on $N$, if $g^{*} \eta=\eta$, i.e. $G$ preserves the contact form, not only the contact structure. A $G$-action by strong contactomorphisms on $(N, \eta)$ admits an equivariant momentum map $J: N \rightarrow \mathfrak{g}^{*}$ given by evaluating the contact form on fundamental fields: $\langle J, \xi\rangle=\eta\left(\xi_{N}\right)$.

Throughout this paper we shall denote by $\mathfrak{g}$ the Lie algebra of $G$, by $\langle\cdot, \cdot\rangle: \mathfrak{g}^{*} \times \mathfrak{g} \rightarrow \mathbb{R}$ the natural pairing between $\mathfrak{g}^{*}$ and $\mathfrak{g}$, and by $\xi_{N}$ the fundamental vector field (or infinitesimal generator) defined by $\xi \in \mathfrak{g}$. For simplicity, we shall work exclusively with free proper actions, although the extensions of our results to locally free actions is routine; in that case the relevant quotient spaces will be orbifolds instead of manifolds. For a smooth map $f: A \rightarrow B$ between the manifolds $A$ and $B, T_{a} f: T_{a} A \rightarrow T_{f(a)} B$ denotes its derivative, or tangent map, at $a \in A$. 
The momentum map $J$ is constant on the flow of the Reeb vector field. In addition,

$$
\left\langle T_{n} J(v), \xi\right\rangle=d \eta(n)\left(v, \xi_{N}(n)\right)
$$

for any $n \in N, v \in T_{n} N$, and $\xi \in \mathfrak{g}$. This immediately implies

$$
\left[\operatorname{im}\left(T_{n} J\right)\right]^{\circ}=\left\{\xi \in \mathfrak{g} \mid d \eta(n)\left(\xi_{N}(n), \cdot\right)=0\right\},
$$

which is the contact analogue of the bifurcation lemma from the usual theory of momentum maps on Poisson manifolds; the term on the left is the annihilator of the subspace in parentheses. For this (contact) momentum map, $0 \in \mathfrak{g}^{*}$ is a regular value if and only if the fundamental fields induced by the action do not vanish on the zero level set of $J$. Moreover, if this is the case, the pull back of the contact form to $J^{-1}(0)$ is basic. Let $\pi_{0}: J^{-1}(0) \rightarrow J^{-1}(0) / G$ and $\iota_{0}: J^{-1}(0) \hookrightarrow N$ be the canonical projection and inclusion respectively. The reduction theorem asserts the existence of a unique contact form $\eta_{0}$ on $J^{-1}(0) / G$ such that $\pi_{0}^{*} \eta_{0}=\iota_{0}^{*} \eta$.

Regarding contact reduction at $\mu \neq 0$, up to now there are two versions available: one due to Albert [2] and a very recent one due to Willett [11].

Albert's method [2]. Let $(N, \eta)$ be an exact contact manifold with Reeb vector field $R$ and let $\Phi$ be a "good" action of a Lie group by strong contactomorphisms. For $\mu \in \mathfrak{g}^{*}$, denote by $G_{\mu}$ the isotropy group at $\mu$ of the coadjoint action and by $\mathfrak{g}_{\mu}$ its Lie algebra. If $\mu \neq 0$ is a regular value of $J$ the restriction of the contact form to $J^{-1}(\mu)$ is not basic. This problem is overcome by Albert by changing the infinitesimal action of $\mathfrak{g}_{\mu}$ on $J^{-1}(\mu)$ as follows: $\xi \mapsto \xi_{N}-\langle\mu, \xi\rangle R$, where $R$ is the Reeb vector field. In general, this infinitesimal action cannot be integrated to an action of $G_{\mu}$. However, if $R$ is complete, this $\mathfrak{g}_{\mu}$-action is induced by an action of the universal covering group $\widehat{G}_{\mu}$ (if $G_{\mu}$ is connected) given by

$$
\left(e^{t \xi}, n\right) \mapsto \phi_{e^{t \xi}}\left(\rho_{t\langle\mu, \xi\rangle}^{-1}(n)\right),
$$

where $\rho_{t}$ is the flow of the Reeb vector field. Albert defines the reduced space as $J^{-1}(\mu) / \widehat{G}_{\mu}$ via this new action and shows it is naturally a contact manifold.

Willett's method [11]. The idea is to expand $\mu$ and to shrink $G_{\mu}$. As above, $G$ is a Lie group that acts smoothly on an exact contact manifold $(N, \eta)$ preserving the contact form $\eta$. Let $\mu \in \mathfrak{g}^{*}$. Willett calls the kernel group of $\mu$, the connected Lie subgroup $K_{\mu}$ of $G_{\mu}$ with 
Lie algebra $\mathfrak{k}_{\mu}=\operatorname{ker}\left(\left.\mu\right|_{\mathfrak{g}_{\mu}}\right)$. It is easy to see that $\mathfrak{k}_{\mu}$ is an ideal in $\mathfrak{g}_{\mu}$ and therefore $K_{\mu}$ is a connected normal subgroup of $G_{\mu}$. Contact reduction (or the contact quotient) of $N$ by $G$ at $\mu$ is defined by Willett as

$$
N_{\mu}:=J^{-1}\left(\mathbb{R}_{+} \mu\right) / K_{\mu} \text {. }
$$

Assume that $K_{\mu}$ acts freely and properly on $J^{-1}\left(\mathbb{R}_{+} \mu\right)$. Then $J$ is transversal to $\mathbb{R}_{+} \mu$ and the pull back of $\eta$ to $J^{-1}\left(\mathbb{R}_{+} \mu\right)$ is basic relative to the $K_{\mu}$-action on $J^{-1}\left(\mathbb{R}_{+} \mu\right)$ and thus induces a one form $\eta_{\mu}$ on the quotient $N_{\mu}$. If, in addition, $\operatorname{ker} \mu+\mathfrak{g}_{\mu}=\mathfrak{g}$ then the form $\eta_{\mu}$ is also a contact form. It is characterized, as usual, by the identity $\pi_{\mu}^{*} \eta_{\mu}=i_{\mu}^{*} \eta$, where $\pi_{\mu}: J^{-1}\left(\mathbb{R}_{+} \mu\right) \rightarrow N_{\mu}$ is the canonical projection and $i_{\mu}: J^{-1}\left(\mathbb{R}_{+} \mu\right) \hookrightarrow N$ is the canonical inclusion.

It is to be noted that for $\mu=0$, Albert's and Willett's quotients coincide because in Albert's method for $\mu=0$ the contact form restricted to $J^{-1}(0)$ is basic and thus there is no need to change the infinitesimal action. Hence both reduction methods yield in this case the space $J^{-1}(0) / G$.

Notations: Throughout the paper we shall denote by $\pi_{G}: Q \rightarrow Q / G$, $\pi_{Q / G}: T^{*}(Q / G) \rightarrow Q / G, \pi_{Q}: T^{*} Q \rightarrow Q$ the respective canonical projections. The Liouville one-forms of $T^{*} Q$ and $T^{*}(Q / G)$ will be denoted respectively by $\theta$ and $\Theta$. The naturally lifted action of $G$ on $T^{*} Q$ admits an equivariant momentum map $J_{c t}: T^{*} Q \rightarrow \mathfrak{g}^{*}$ given by $\left\langle J_{c t}\left(\alpha_{q}\right), \xi\right\rangle=\alpha_{q}\left(\xi_{Q}(q)\right)$, where $\alpha_{q} \in T_{q}^{*} Q, \xi \in \mathfrak{g}$, and $\xi_{Q}$ denotes the fundamental vector field defined by the $G$-action on $Q$.

\section{The cosphere bundle and its contact structure}

Let $Q$ be a differentiable manifold of real dimension $n, \pi_{Q}: T^{*} Q \rightarrow Q$ its cotangent bundle, and $\theta$ the Liouville form on $T^{*} Q$. We shall denote by $\alpha_{q}, \beta_{q}$ etc. the elements of $T^{*} Q$.

Let $G$ be a finite dimensional Lie subgroup of $\operatorname{Diff}(Q)$ and denote by $\Phi: G \times Q \rightarrow Q$ a free, proper action of $G$ on $Q$. We denote by $\Phi_{*}: G \times T^{*} Q \rightarrow T^{*} Q$ its natural lift to the cotangent bundle of $Q$. $\Phi_{*}$ is still free and proper and preserves the Liouville form $\theta$ and thus the canonical symplectic structure $-d \theta$ of $T^{*} Q$.

Consider the action of the multiplicative group $\left.\mathbb{R}_{+}=\right] 0,+\infty[$ by dilations on the fibers of $T^{*} Q \backslash\{0\}$.

Definition 2.1. The cosphere bundle $S^{*} Q$ of $Q$ is the quotient manifold $\left(T^{*} Q \backslash\{0\}\right) / \mathbb{R}_{+}$. Denote by $\kappa:\left[\alpha_{q}\right] \in S^{*} Q \mapsto q \in Q$ the associated canonical projection. 
The construction described below is standard (see e.g. $[\mathbf{9}]$ ).

Let $\pi: T^{*} Q \backslash\{0\} \rightarrow S^{*} Q$ be the canonical projection. The elements of the cosphere bundle are classes that we denote with $\left[\alpha_{q}\right]$. Of course, $\left(\pi, \mathbb{R}_{+}, T^{*} Q \backslash\{0\}, S^{*} Q\right)$ is a $\mathbb{R}_{+}$-principal bundle. As such, it always has global sections: it is enough to choose a Riemannian metric on $Q$ (supposed paracompact), to identify $T^{*} Q$ with $T Q, S^{*} Q$ with the unit sphere bundle $T^{1} Q$ of $T Q$, and to consider the canonical inclusion $T^{1} Q \hookrightarrow T Q$. Let then $\sigma: S^{*} Q \rightarrow T^{*} Q \backslash\{0\}$ be a global section. The equation

$$
\sigma \circ \pi=f_{\sigma} 1_{T^{*} Q \backslash\{0\}}
$$

where $1_{T^{*} Q \backslash\{0\}}$ denotes the identity map of $T^{*} Q \backslash\{0\}$, defines a function $f_{\sigma}: T^{*} Q \backslash\{0\} \rightarrow \mathbb{R}_{+}$with the following property of compatibility with respect to the action of $\mathbb{R}_{+}$:

$$
f_{\sigma}\left(r \alpha_{q}\right)=\frac{1}{r} f_{\sigma}\left(\alpha_{q}\right), \quad r \in \mathbb{R}_{+}, \alpha_{q} \in T^{*} Q \backslash\{0\} .
$$

Indeed, $\sigma\left(\left[\alpha_{q}\right]\right)=f_{\sigma}\left(\alpha_{q}\right) \alpha_{q}=\sigma\left(\left[r \alpha_{q}\right]\right)=f_{\sigma}\left(r \alpha_{q}\right) r \alpha_{q}$. The following statement is now clear.

Lemma 2.1. The set of global sections of $\pi: T^{*} Q \backslash\{0\} \rightarrow S^{*} Q$ is in bijective correspondence with the set of $\mathcal{C}^{\infty}$ functions $f: T^{*} Q \backslash\{0\} \rightarrow$ $\mathbb{R}_{+}$satisfying (2.1).

We pull back by $\sigma$ the restriction of the Liouville form and obtain the one-form $\theta_{\sigma}=\sigma^{*} \theta$ on $S^{*} Q$. One has:

$$
\pi^{*} \theta_{\sigma}=f_{\sigma} \theta \text {. }
$$

Indeed, $\pi^{*} \theta_{\sigma}=\pi^{*} \sigma^{*} \theta=(\sigma \circ \pi)^{*} \theta=\left(f_{\sigma} 1_{T^{*} Q \backslash\{0\}}\right)^{*} \theta=f_{\sigma} \theta$. Now, for another global section $\rho$, with associated function $f_{\rho}$, we have

$$
\theta_{\sigma}=\sigma^{*} \theta=(\sigma \circ \pi \circ \rho)^{*} \theta=\rho^{*} \pi^{*} \theta_{\sigma}=\rho^{*}\left(f_{\sigma} \theta\right)=\left(f_{\sigma} \circ \rho\right) \theta_{\rho},
$$

and hence we obtain

$$
\theta_{\sigma}=g_{\sigma \rho} \theta_{\rho}, \quad \text { with } \quad g_{\sigma \rho}=f_{\sigma} \circ \rho .
$$

Note also that $g_{\sigma \rho} \circ \pi=f_{\sigma} / f_{\rho}$. From (2.3) we easily derive that $\theta_{\sigma}$ is a contact form on $S^{*} Q$ if and only if $\theta_{\rho}$ is one. But it was proved in [1] that if $\sigma$ is defined using a Riemannian metric on $Q$, as explained above, then $\theta_{\sigma}$ is a contact form. Thus we have proved:

Lemma 2.2. $\theta_{\sigma}$ is a global contact form on $S^{*} Q$ for any global section $\sigma$. 
It is also clear from (2.3) that all these contact forms have the same null space, so that the contact structure does not depend on the choice of $\sigma$.

Remark 2.1. Let $\mathcal{C}\left(S^{*} Q\right)=S^{*} Q \times \mathbb{R}_{+}$be the symplectic cone over $S^{*} Q$, endowed with the symplectic form $d\left(t \theta_{\sigma}\right)$. Then one can easily see that $T_{\sigma}: \mathcal{C}\left(S^{*} Q\right) \rightarrow T^{*} Q$ given by $T_{\sigma}\left(\left[\alpha_{q}\right], t\right)=t f_{\sigma}\left(\alpha_{q}\right) \cdot \alpha_{q}$ is a well defined symplectic diffeomorphism, that is, a symplectomorphism.

\section{The reduction theorems}

We shall now lift the free proper action of $G$ to the cosphere bundle and compute the associated momentum map. The action $\Phi$ lifts to an action $\Phi_{*}$ on $T^{*} Q$ by setting

$$
\Phi_{*}\left(g, \alpha_{q}\right):=T_{\Phi(g, q)}^{*} \Phi_{g^{-1}} \alpha_{q},
$$

for $g \in G, \alpha_{q} \in T_{q}^{*} Q$, and where the upper star denotes the dual map of the linear map to which it is applied. It is clear that the cotangent bundle projection $\pi_{Q}: T^{*} Q \rightarrow Q$ is equivariant relative to the actions $\Phi_{*}$ and $\Phi$. If the action $\Phi$ is free and proper, this equivariance immediately shows that the action $\Phi_{*}$ is also free and proper.

Denote by $\kappa_{Q}:\left[\alpha_{q}\right] \in S^{*} Q \mapsto q \in Q$ the canonical cosphere bundle projection.

Lemma 3.1. The action $\Phi$ induces a free proper action $\widehat{\Phi}_{*}: G \times S^{*} Q \rightarrow$ $S^{*} Q$.

Proof. Define

$$
\widehat{\Phi}_{*}\left(g,\left[\alpha_{q}\right]\right)=\left[\Phi_{*}\left(g, \alpha_{q}\right)\right] .
$$

As $\widehat{\Phi}_{*}\left(g,\left[r \alpha_{q}\right]\right)=\left[\Phi_{*}\left(g, r \alpha_{q}\right)\right]=\left[r \Phi_{*}\left(g, \alpha_{q}\right)\right]=\left[\Phi_{*}\left(g, \alpha_{q}\right)\right]$, the definition is correct. Note also that $\Phi_{*}$ covers $\Phi$, that is, $\kappa_{Q} \circ \widehat{\Phi}_{*}=\Phi \circ \kappa_{Q}$. This immediately proves that freeness (respectively properness) of the $G$ action on $Q$ implies freeness (respectively properness) of the action $\widehat{\Phi}_{*}$ on $S^{*} Q$. Clearly $\left(\widehat{\Phi}_{* g}\right)^{*} \theta_{\sigma}$ is a multiple of $\theta_{\sigma}$ and the proof is complete.

Lemma 3.2. The action $\widehat{\Phi}_{*}: G \times S^{*} Q \rightarrow S^{*} Q$ is by contactomorphisms and the scale factors are all positive. 
Proof. By direct computation and using (2.2) in the last equality, we have:

$$
\begin{aligned}
\widehat{\Phi}_{* g}^{*} \theta_{\sigma}\left(\left[\alpha_{q}\right]\right)\left(v_{\left[\alpha_{q}\right]}\right) & =\theta_{\sigma}\left(\widehat{\Phi}_{* g}\left(\left[\alpha_{q}\right]\right)\right)\left(T_{\left[\alpha_{q}\right]} \widehat{\Phi}_{* g}\left(v_{\left[\alpha_{q}\right]}\right)\right) \\
& =\theta\left(\left(\sigma \circ \widehat{\Phi}_{* g}\right)\left(\left[\alpha_{q}\right]\right)\right)\left(T_{\left[\alpha_{q}\right]}\left(\sigma \circ \widehat{\Phi}_{* g}\right)\left(v_{\left[\alpha_{q}\right]}\right)\right) \\
& =\left(\sigma \circ \widehat{\Phi}_{* g}\right)\left(\left[\alpha_{q}\right]\right)\left(T_{\left[\alpha_{q}\right]}\left(\pi_{Q} \circ \sigma \circ \widehat{\Phi}_{* g}\right)\left(v_{\left[\alpha_{q}\right]}\right)\right) \\
& =f_{\sigma}\left(\Phi_{* g}\left(\alpha_{q}\right)\right) \Phi_{* g}\left(\alpha_{q}\right)\left(T_{\left[\alpha_{q}\right]}\left(\pi_{Q} \circ \sigma \circ \widehat{\Phi}_{* g}\right)\left(v_{\left[\alpha_{q}\right]}\right)\right) \\
& =f_{\sigma}\left(\Phi_{* g}\left(\alpha_{q}\right)\right) \alpha_{q}\left(T_{\left[\alpha_{q}\right]}\left(\Phi_{g}^{-1} \circ \pi_{Q} \circ \sigma \circ \widehat{\Phi}_{* g}\right)\left(v_{\left[\alpha_{q}\right]}\right)\right) \\
& =f_{\sigma}\left(\Phi_{* g}\left(\alpha_{q}\right)\right) \alpha_{q}\left(T_{\left[\alpha_{q}\right]}\left(\pi_{Q} \circ \sigma\right)\left(v_{\left[\alpha_{q}\right]}\right)\right) \\
& =f_{\sigma}\left(\Phi_{* g}\left(\alpha_{q}\right)\right) \theta\left(\alpha_{q}\right)\left(T_{\left[\alpha_{q}\right]}(\sigma)\left(v_{\left[\alpha_{q}\right]}\right)\right) \\
& =\frac{f_{\sigma}\left(\Phi_{* g}\left(\alpha_{q}\right)\right)}{f_{\sigma}\left(\alpha_{q}\right)} \theta_{\sigma}\left(\left[\alpha_{q}\right]\right)\left(v_{\left[\alpha_{q}\right]}\right) .
\end{aligned}
$$

To construct a momentum map associated to this action, we need to work with a strong action, that is, we need it to preserve not only the contact structure, but the contact form. This can be achieved by adapting Palais' argument (or, if $G$ is compact, by averaging). Indeed, owing to Lemma 3.1, we may apply Proposition 2.8 in [5] asserting that for a proper action by contactomorphisms, there always exist an invariant contact form. (The proof of this is a straightforward modification of the classical proof of Palais for the existence of invariant Riemannian metrics on paracompact manifolds endowed with a proper Lie group action.) As every contact form on the cosphere bundle is obtained via a global section as above, we shall chose once and for all a section $\sigma$ for which $\left(\widehat{\Phi}_{* g}\right) * \theta_{\sigma}=\theta_{\sigma}$. Relative to this contact form the induced action on the cosphere bundle is by strong contactomorphisms.

The associated momentum map $J_{\theta_{\sigma}}$ will be denoted for simplicity by $J$ since in what follows no other contact form different from $\theta_{\sigma}$ will be used. Let $\left(S^{*} Q\right)_{0}=J^{-1}(0) / G$ be the reduced space corresponding to the regular value $0 \in \mathfrak{g}^{*}$.

Similar considerations apply to the manifold $Q / G$ proving that its cosphere bundle is a contact manifold. As above, the contact structure can be described as the kernel of a contact form of the type $\Theta_{\Sigma}$, where $\Sigma: S^{*}(Q / G) \rightarrow T^{*}(Q / G) \backslash\{0\}$ is a global section and $\Theta$ is the Liouville form of $T^{*}(Q / G)$. 
Theorem 3.1. Let $G$ be a Lie group acting freely and properly on the manifold $Q$. Then $\left(S^{*} Q\right)_{0}$, the reduced space at the regular value zero of the momentum map of the cosphere bundle of $Q$, is contact-diffeomorphic with the cosphere bundle $S^{*}(Q / G)$.

Remark 3.1. Suppose $(N, \eta)$ is a contact manifold on which a Lie group $G$ acts by strong contactomorphisms. The action can be naturally lifted to the symplectic cone $(\mathcal{C}(N), d(t \eta))$ by letting $G$ act trivially on $\mathbb{R}_{+}$; one obtains an action by symplectomorphisms. It is well known that, in this situation, the reduced symplectic space at 0 is the symplectic cone over the contact reduced space at $0: \mathcal{C}\left(N_{0}\right) \cong(\mathcal{C}(N))_{0}$. This can be applied to $N=S^{*} Q$ and combined with the cotangent bundle reduction theorem it should lead to a "diagram chasing" proof of the theorem. However, we prefer to make the maps involved in the proof precise.

Proof. A first key observation is that the actions of $G$ and $\mathbb{R}_{+}$on $T^{*} Q \backslash$ $\{0\}$ commute, so that there exists the diffeomorphism:

$$
\lambda:\left(S^{*} Q\right) / G=\left(T^{*} Q \backslash\{0\} / \mathbb{R}_{+}\right) / G \rightarrow\left(T^{*} Q \backslash\{0\} / G\right) / \mathbb{R}_{+} .
$$

Second, applying the cotangent bundle reduction theorem to $T^{*} Q$, we have the symplectic diffeomorphism (see [1], [6], or [8])

$$
\varphi_{0}: J_{c t}^{-1}(0) / G \rightarrow T^{*}(Q / G), \quad \text { given by } \varphi_{0}\left(\widehat{\alpha}_{q}\right)\left(T_{q} \pi_{G}\left(v_{q}\right)\right):=\alpha_{q}\left(v_{q}\right) \text {, }
$$

where $v_{q} \in T_{q} Q, \alpha_{q} \in J_{c t}^{-1}(0) \cap T_{q}^{*} Q, \widehat{\alpha}_{q} \in J_{c t}^{-1}(0) / G$ is its class in the reduced space at zero, and $\pi_{G}: Q \rightarrow Q / G$ is the projection. Denote by $p_{0}: J_{c t}^{-1}(0) \rightarrow J_{c t}^{-1}(0) / G$ the canonical projection, that is, $p_{0}\left(\alpha_{q}\right)=\widehat{\alpha}_{q}$ for all $\alpha_{q} \in J_{c t}^{-1}(0)$.

We want to relate the zero level sets of the contact momentum map $J$ and of the symplectic momentum map $J_{c t}$. The definition of the (contact) momentum map $J$, the $\pi$ relatedness of $\xi_{T^{*} Q}$ and $\xi_{S^{*} Q}$, formula (2.2), the definition of the Liouville form on $T^{*} Q$, and finally the $\pi_{Q}$ relatedness of $\xi_{T^{*} Q}$ and $\xi_{Q}$ yield for any $\xi \in \mathfrak{g}$

$$
\begin{aligned}
\left\langle J\left(\left[\alpha_{q}\right]\right), \xi\right\rangle & =\theta_{\sigma}\left(\left[\alpha_{q}\right]\right)\left(\xi_{S^{*} Q}\left(\left[\alpha_{q}\right]\right)\right) \\
& =\theta_{\sigma}\left(\pi\left(\alpha_{q}\right)\right)\left(T_{\alpha_{q}} \pi\left(\xi_{T^{*} Q}\left(\alpha_{q}\right)\right)\right. \\
& =\left(\pi^{*} \theta_{\sigma}\right)\left(\alpha_{q}\right)\left(\xi_{T^{*} Q}\left(\alpha_{q}\right)\right) \\
& =f_{\sigma}\left(\alpha_{q}\right) \theta\left(\alpha_{q}\right)\left(\xi_{T^{*} Q}\left(\alpha_{q}\right)\right) \\
& =f_{\sigma}\left(\alpha_{q}\right) \alpha_{q}\left(T_{\alpha_{q}} \pi_{Q}\left(\xi_{T^{*} Q}\left(\alpha_{q}\right)\right)\right) \\
& =f_{\sigma}\left(\alpha_{q}\right) \alpha_{q}\left(\xi_{Q}(q)\right),
\end{aligned}
$$


that is,

$$
J\left(\left[\alpha_{q}\right]\right)=f_{\sigma}\left(\alpha_{q}\right) \alpha_{q} .
$$

Since $f_{\sigma}>0$, this implies that

$$
J^{-1}(0)=\left\{\left[\alpha_{q}\right] \mid \alpha_{q}\left(\xi_{Q}(q)\right)=0, \text { for all } \xi \in \mathfrak{g}\right\} .
$$

However, $\left\langle J_{c t}\left(\alpha_{q}\right), \xi\right\rangle=\alpha_{q}\left(\xi_{Q}(q)\right)$ for any $\xi \in \mathfrak{g}$, which shows that $J^{-1}(0) \subseteq \pi\left(J_{c t}^{-1}(0)\right)$. The converse inclusion being obvious, we conclude that $J^{-1}(0)=\pi\left(J_{c t}^{-1}(0)\right)$ and hence

$$
\left(S^{*} Q\right)_{0}:=J^{-1}(0) / G=\left(J_{c t}^{-1}(0) \backslash\{0\} / \mathbb{R}_{+}\right) / G .
$$

Denote by

$$
\bar{\lambda}:\left(S^{*} Q\right)_{0} \rightarrow\left(J_{c t}^{-1}(0) \backslash\{0\} / G\right) / \mathbb{R}_{+}
$$

the diffeomorphism obtained by restricting the diffeomorphism $\lambda$ defined in (3.1) to $\left(S^{*} Q\right)_{0}$ and denote by $\bar{\theta}_{\sigma}$ the reduced contact form on $\left(S^{*} Q\right)_{0}$.

The definition of the diffeomorphism $\varphi_{0}: J_{c t}^{-1}(0) / G \rightarrow T^{*}(Q / G)$ defined in (3.2) shows that $\widehat{0}_{q} \in J_{c t}^{-1}(0) / G$ is mapped to the zero element of $T_{\pi_{G}(q)}^{*}(Q / G)$ and that $\varphi_{0}$ commutes with the $\mathbb{R}_{+}$-actions on $J_{c t}^{-1}(0) / G$ and on $T^{*}(Q / G)$ respectively. Thus $\varphi_{0}$ induces a smooth map

$$
\widehat{\varphi}_{0}:\left(J_{c t}^{-1}(0) \backslash\{0\} / G\right) / \mathbb{R}_{+} \rightarrow\left(T^{*}(Q / G) \backslash\{0\}\right) / \mathbb{R}_{+}=S^{*}(Q / G)
$$

given by

$$
\widehat{\varphi}_{0}\left(\left[\widehat{\alpha}_{q}\right]\right):=\left[\varphi_{0}\left(\widehat{\alpha}_{q}\right)\right],
$$

where $\left[\widehat{\alpha}_{q}\right] \in\left(J_{c t}^{-1}(0) / G\right) / \mathbb{R}_{+}$denotes the class of $\widehat{\alpha}_{q} \in J_{c t}^{-1}(0) / G$. The same reasoning applied to $\varphi_{0}^{-1}$ shows that it induces a smooth map $S^{*}(Q / G) \rightarrow\left(J_{c t}^{-1}(0) \backslash\{0\} / G\right) / \mathbb{R}_{+}$which is easily verified to be the inverse of $\widehat{\varphi}_{0}$, that is, $\widehat{\varphi}_{0}$ is a diffeomorphism.

The theorem will be proved if it is shown that $\widehat{\varphi}_{0} \circ \bar{\lambda}:\left(S^{*} Q\right)_{0} \rightarrow$ $S^{*}(Q / G)$ is a contactomorphism. Let $\Sigma: S^{*}(Q / G) \rightarrow T^{*}(Q / G) \backslash\{0\}$ be a global section and let $\Theta_{\Sigma}:=\Sigma^{*} \Theta$ be the contact form on $S^{*}(Q / G)$ associated to this section, where $\Theta$ is the Liouville form on $T^{*}(Q / G)$. From the discussion in Section 2, we know that $\Theta_{\Sigma}$ is one of the possible contact forms underlying the contact structure of the cosphere bundle $S^{*}(Q / G)$. Thus, to show that $\widehat{\varphi}_{0} \circ \bar{\lambda}$ is a contactomorphism, it will be enough to verify that $\left(\widehat{\varphi}_{0} \circ \bar{\lambda}\right)^{*} \Theta_{\Sigma}$ is proportional to $\bar{\theta}_{\sigma}$, the proportionality factor being a strictly positive function on $\left(S^{*} Q\right)_{0}$. To this end, let $\pi_{0}: J^{-1}(0) \rightarrow J^{-1}(0) / G=\left(S^{*} Q\right)_{0}, \iota_{0}: J^{-1}(0) \hookrightarrow S^{*} Q$ 
be the canonical projection and the canonical inclusion, respectively. From the contact reduction theorem at zero (reviewed in the Introduction), we know that $\bar{\theta}_{\sigma}$ is characterized by the relation $\pi_{0}^{*} \bar{\theta}_{\sigma}=\iota_{0}^{*} \theta_{\sigma}$. Thus, it suffices to show that $\left(\widehat{\varphi}_{0} \circ \bar{\lambda} \circ \pi_{0}\right)^{*} \Theta_{\Sigma}$ is proportional to $\iota_{0}^{*} \theta_{\sigma}$ with a strictly positive function on $J^{-1}(0)$ as proportionality factor.

The commutative diagram below is needed in the proof that follows. All vertical arrows are projections. The maps in this diagram have all been defined with the exception of $\Pi: T^{*}(Q / G) \backslash\{0\} \rightarrow S^{*}(Q / G)$ which is the cosphere bundle projection associated to the manifold $Q / G$ and $\bar{\pi}:\left(J_{c t}^{-1}(0) \backslash\{0\}\right) / G \rightarrow\left(\left(J_{c t}^{-1}(0) \backslash\{0\}\right) / G\right) / \mathbb{R}_{+}$which is associates to each point in $\left(J_{c t}^{-1}(0) \backslash\{0\}\right) / G$ its $\mathbb{R}_{+}$-orbit.

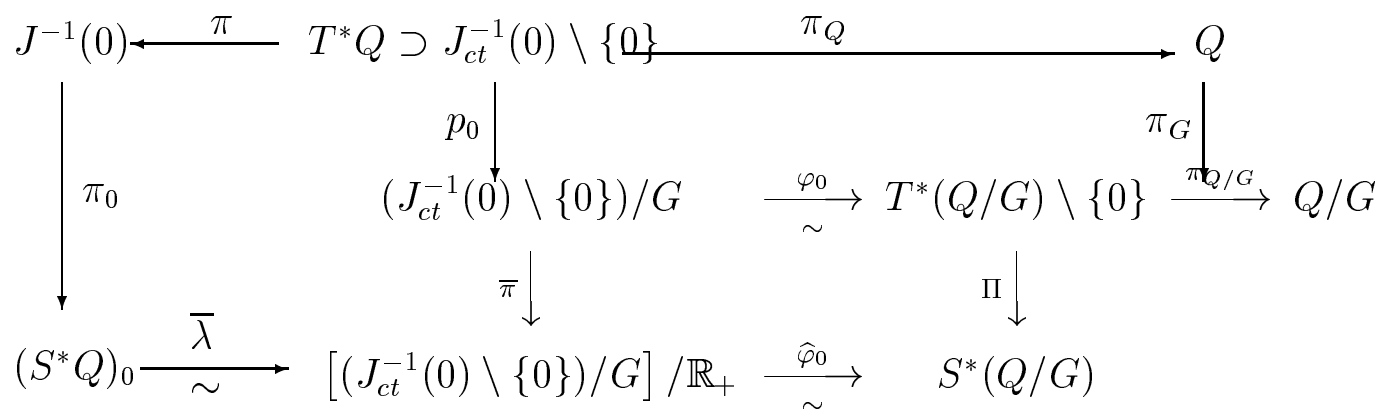

We begin with the computation of $\left(\widehat{\varphi}_{0} \circ \bar{\lambda} \circ \pi_{0} \circ \pi\right)^{*} \Theta_{\Sigma}$. From the commutative diagram we have

$$
\widehat{\varphi}_{0} \circ \bar{\lambda} \circ \pi_{0} \circ \pi=\Pi \circ \varphi_{0} \circ p_{0} \text { and } \pi_{Q / G} \circ \varphi_{0} \circ p_{0}=\pi_{G} \circ \pi_{Q}
$$

so that using (2.2) with base manifold $Q / G$, the definition (3.2) of $\varphi_{0}$, and the global formula of the Liouville form on $T^{*}(Q / G)$, we get for any $\alpha_{q} \in J_{c t}^{-1}(0) \backslash\{0\}$ and any $v \in T_{\alpha_{q}}\left(J_{c t}^{-1}(0) \backslash\{0\}\right)$

$$
\begin{aligned}
\left(\left(\widehat{\varphi}_{0} \circ \bar{\lambda} \circ \pi_{0} \circ \pi\right)^{*} \Theta_{\Sigma}\right)\left(\alpha_{q}\right)(v)=\left(\left(\Pi \circ \varphi_{0} \circ p_{0}\right)^{*} \Theta_{\Sigma}\right)\left(\alpha_{q}\right)(v) \\
\quad=\left(\left(\varphi_{0} \circ p_{0}\right)^{*}\left(\Pi^{*} \Theta_{\Sigma}\right)\right)\left(\alpha_{q}\right)(v)=\left(\left(\varphi_{0} \circ p_{0}\right)^{*}\left(f_{\Sigma} \Theta\right)\right)\left(\alpha_{q}\right)(v) \\
\quad=\left(f_{\Sigma} \circ \varphi_{0} \circ p_{0}\right)\left(\alpha_{q}\right)\left(\varphi_{0} \circ p_{0}\right)\left(\alpha_{q}\right)\left(T_{\alpha_{q}}\left(\pi_{Q / G} \circ \varphi_{0} \circ p_{0}\right)(v)\right) \\
\quad=\left(f_{\Sigma} \circ \varphi_{0} \circ p_{0}\right)\left(\alpha_{q}\right)\left(\varphi_{0} \circ p_{0}\right)\left(\alpha_{q}\right)\left(T_{\alpha_{q}}\left(\pi_{G} \circ \pi_{Q}\right)(v)\right) \\
\quad=\left(f_{\Sigma} \circ \varphi_{0} \circ p_{0}\right)\left(\alpha_{q}\right) \alpha_{q}\left(T_{\alpha_{q}} \pi_{Q}(v)\right) .
\end{aligned}
$$


On the other hand, since $\iota_{0}: J^{-1}(0) \hookrightarrow S^{*} Q$ is the inclusion, from (2.2) and the definition of the Liouville form on $T^{*} Q$, we get

$$
\left(\left(\iota_{0} \circ \pi\right)^{*} \theta_{\sigma}\right)\left(\alpha_{q}\right)(v)=\left(f_{\sigma} \theta\right)\left(\alpha_{q}\right)(v)=f_{\sigma}\left(\alpha_{q}\right) \alpha_{q}\left(T_{\alpha_{q}} \pi_{Q}(v)\right) .
$$

The two identities (3.8) and (3.9) show that on $J_{c t}^{-1}(0) \backslash\{0\}$ we have the equality

$$
\pi^{*}\left(\widehat{\varphi}_{0} \circ \bar{\lambda} \circ \pi_{0}\right)^{*} \Theta_{\Sigma}=\frac{f_{\Sigma} \circ \varphi_{0} \circ p_{0}}{f_{\sigma}} \pi^{*} \iota_{0}^{*} \theta_{\sigma} .
$$

Formula (2.1) shows that the strictly positive proportionality factor in (3.10) drops to a strictly positive function $F$ on the quotient $J^{-1}(0)$. Since $\pi$ is a surjective submersion, (3.10) implies that $\left(\widehat{\varphi}_{0} \circ \bar{\lambda} \circ \pi_{0}\right)^{*} \Theta_{\Sigma}=$ $F \iota_{0}^{*} \theta_{\sigma}$ where the function $F>0$, which is the desired identity.

The first two examples below use parallelizable manifolds $Q$. Note that for an $n$-dimensional parallelizable manifold $Q$, the cosphere bundle is $S^{*} Q=Q \times S^{n-1}$.

Example 3.1. Let $Q=\mathbb{T}^{n}$ and $G=S^{1}$ acting by multiplication on the first factor of the torus and trivially on the other ones. Then $Q / G=\mathbb{T}^{n-1}$ and $S^{*}\left(\mathbb{T}^{n}\right)=\mathbb{T}^{n} \times S^{n-1}$. Hence, by Theorem 3.1, we find that $\left(\mathbb{T}^{n} \times S^{n-1}\right)_{0}$ is contactomorphic with $\mathbb{T}^{n-1} \times S^{n-2}$.

Example 3.2. Let $Q=\mathbb{R}^{n}$ and $G=\mathbb{Z}^{n}$ acting by translations on each factor. Then $Q / G=\mathbb{T}^{n}, S^{*}(Q / G)=\mathbb{T}^{n} \times S^{n-1}, S^{*}\left(\mathbb{R}^{n}\right)=\mathbb{R}^{n} \times S^{n-1}$, hence we obtain the contactomorphism $\left(\mathbb{R}^{n} \times S^{n-1}\right)_{0} \cong \mathbb{T}^{n} \times S^{n-1}$.

Example 3.3. Let $Q=S^{3}$ and $G=S^{1}$ acting by multiplication (of unitary quaternions by unit complex numbers). Then $Q / G=S^{2}$, the base of the Hopf fibration. It is well known (see, e.g. [7], Exercise 1.2-4) that $S^{*}\left(S^{2}\right)$ is diffeomorphic with $S O(3)$. On the other hand, $S^{*}\left(S^{3}\right)=S^{2} \times S^{3}$. We thus obtain the contact diffeomorphism $\left(S^{2} \times\right.$ $\left.S^{3}\right)_{0} \cong S O(3)$.

If we want to carry out the cosphere bundle reduction at a point $\mu \neq$ 0, we have a priori two choices: to use Albert's or Willett's reduction methods.

Regarding Albert's reduction method (see its description in the Introduction), nothing will guarantee that the action of the universal cover $\widehat{G}_{\mu}$ on $S^{*} Q$ is induced by an action on $Q$. Example II in [2] describes precisely such a situation. It refers (without naming it explicitly) to the cosphere bundle $S^{*} \mathbb{T}^{n}$ of the $n$-dimensional torus $\mathbb{T}^{n}$. 
The group is $G=\mathbb{T}^{n}$ which acts trivially on itself. For a non-zero regular value $\mu$ of norm 1 , Albert applies his construction with $\widehat{G}_{\mu}=\mathbb{R}^{n}$ and obtains the standard circle $S^{1}$ as the reduced space. But the action of $\mathbb{R}^{n}$ on $S^{*} \mathbb{T}^{n}$ does not come from an action of $\mathbb{R}^{n}$ on $T^{n}$ ! Thus, Albert's method cannot be used to do contact reduction of the cosphere bundle at a non zero value of the momentum map.

However, Willett's method can be applied, as we shall show below. Contact reduction at a non zero value of the momentum map will embed in a certain cosphere bundle. The precise statement is the following. Recall that $K_{\mu}$ denotes the connected normal Lie subgroup of $G_{\mu}$ whose Lie algebra is the ideal $\mathfrak{k}_{\mu}:=\operatorname{ker}\left(\left.\mu\right|_{\mathfrak{g}_{\mu}}\right)$ in $\mathfrak{g}_{\mu}$.

Theorem 3.2. Let $Q$ be a differentiable manifold of real dimension $n$, $G$ a finite dimensional Lie subgroup of $\operatorname{Diff}(Q)$ and $\Phi: G \times Q \rightarrow Q$ a smooth action of $G$ on $Q$. Assume that $K_{\mu}$ acts freely and properly on $J^{-1}\left(\mathbb{R}_{+} \mu\right)$ and that $\operatorname{ker} \mu+\mathfrak{g}_{\mu}=\mathfrak{g}$. Then the contact reduction

$$
\left(S^{*} Q\right)_{\mu}=J^{-1}\left(\mathbb{R}_{+} \mu\right) / K_{\mu}
$$

is embedded by a map preserving the contact structures onto a subbundle of $S^{*}\left(Q / K_{\mu}\right)$.

Proof. Consider the cosphere bundle $S^{*} Q$ endowed with the contact form $\theta_{\sigma}$ preserved by the $G$-action. Willett $[\mathbf{1 1}] \S 3$ proves that $J$ is transversal to $\mathbb{R}_{+} \mu$ if and only if the $K_{\mu}$-action on $J^{-1}\left(\mathbb{R}_{+} \mu\right)$ is locally free. Our hypothesis is that this action is in fact free, so the transversality hypothesis in Willett's reduction theorem is satisfied. Together with the other two stated hypotheses, these are precisely the assumptions of Willett's reduction theorem reviewed in the Introduction. Thus $\left(S^{*} Q\right)_{\mu}=J^{-1}\left(\mathbb{R}_{+} \mu\right) / K_{\mu}$ is an exact contact manifold whose contact form, denoted by $\bar{\theta}_{\sigma, \mu}$, is characterized by the identity $\iota_{\mu}^{*} \theta_{\sigma}=\pi_{\mu}^{*} \bar{\theta}_{\sigma, \mu}$, where $\iota_{\mu}: J^{-1}\left(\mathbb{R}_{+} \mu\right) \hookrightarrow S^{*} Q$ is the inclusion and $\pi_{\mu}: J^{-1}\left(\mathbb{R}_{+} \mu\right) \rightarrow J^{-1}\left(\mathbb{R}_{+} \mu\right) / K_{\mu}=\left(S^{*} Q\right)_{\mu}$ is the canonical projection.

As in the proof of Theorem 3.1, $J^{-1}\left(\mathbb{R}_{+} \mu\right)=\pi\left(J_{c t}^{-1}\left(\mathbb{R}_{+} \mu\right)\right)$ and, consequently,

$$
\left(S^{*} Q\right)_{\mu}:=J^{-1}\left(\mathbb{R}_{+} \mu\right) / K_{\mu}=\left(J_{c t}^{-1}\left(\mathbb{R}_{+} \mu\right) \backslash\{0\} / \mathbb{R}_{+}\right) / K_{\mu} .
$$

Since the actions of $K_{\mu}$ (by cotangent lift) and $\mathbb{R}_{+}$(by dilation in each fiber) on $J_{c t}^{-1}\left(\mathbb{R}_{+} \mu\right)$ commute, there is a diffeomorphism

$$
\bar{\lambda}_{\mu}:\left(S^{*} Q\right)_{\mu} \rightarrow\left(J_{c t}^{-1}\left(\mathbb{R}_{+} \mu\right) \backslash\{0\} / K_{\mu}\right) / \mathbb{R}_{+}
$$


characterized by the property

$$
\bar{\lambda} \circ \pi_{\mu} \circ \pi=\bar{\pi}_{\mu} \circ p_{\mu}
$$

where

$$
\bar{\pi}_{\mu}:\left(J_{c t}^{-1}\left(\mathbb{R}_{+} \mu\right) \backslash\{0\}\right) / K_{\mu} \rightarrow\left[\left(J_{c t}^{-1}\left(\mathbb{R}_{+} \mu\right) \backslash\{0\}\right) / K_{\mu}\right] / \mathbb{R}_{+}
$$

and

$$
p_{\mu}: J_{c t}^{-1}\left(\mathbb{R}_{+} \mu\right) \backslash\{0\} \subset T^{*} Q \backslash\{0\} \rightarrow\left(J_{c t}^{-1}\left(\mathbb{R}_{+} \mu\right) \backslash\{0\}\right) / K_{\mu}
$$

are the canonical projections. If $\alpha_{q} \in J_{c t}^{-1}\left(\mathbb{R}_{+} \mu\right) \backslash\{0\} \subset T^{*} Q$, denote by $\widehat{\alpha}_{q}=p_{\mu}\left(\alpha_{q}\right)$ its class in $\left(J_{c t}^{-1}\left(\mathbb{R}_{+} \mu\right) \backslash\{0\}\right) / K_{\mu}$.

Define the map

$$
\psi_{\mu}:\left(J_{c t}^{-1}\left(\mathbb{R}_{+} \mu\right) \backslash\{0\}\right) / K_{\mu} \rightarrow T^{*}\left(Q / K_{\mu}\right) \backslash\{0\}
$$

by

$$
\psi_{\mu}\left(\widehat{\alpha}_{q}\right)\left(T_{q} \pi_{K_{\mu}}\left(v_{q}\right)\right)=\alpha_{q}\left(v_{q}\right)
$$

where $\pi_{K_{\mu}}: Q \rightarrow Q / K_{\mu}$ is the canonical projection. To show that $\psi_{\mu}$ is well defined, observe that for all $\alpha_{q^{\prime}}=T_{\phi(g, q)}^{*} \Phi_{g^{-1}} \alpha_{q}$ with $q^{\prime}=\Phi(g, q)$, $v_{q^{\prime}}=T_{\Phi(g, q)} \Phi_{g}\left(v_{q}+\xi_{Q}(q)\right)$, and $\xi \in \mathfrak{k}_{\mu}$ identity (3.3) implies that

$$
\begin{aligned}
\alpha_{q^{\prime}}\left(v_{q^{\prime}}\right) & =T_{\Phi(g, q)}^{*}\left(\Phi_{g^{-1}} \alpha_{q}, T_{\Phi(g, q)} \Phi_{g}\left(v_{q}+\xi_{Q}(q)\right)\right. \\
& =\alpha_{q}\left(v_{q}+\xi_{Q}(q)\right)=\alpha_{q}\left(v_{q}\right)+\alpha_{q}\left(\xi_{Q}(q)\right) \\
& =\alpha_{q}\left(v_{q}\right)+\frac{1}{f_{\sigma}\left(\alpha_{q}\right)}\left\langle J\left(\left[\alpha_{q}\right]\right), \xi\right\rangle \\
& =\alpha_{q}\left(v_{q}\right)+\frac{1}{f_{\sigma}\left(\alpha_{q}\right)}\langle\mu, \xi\rangle=\alpha_{q}\left(v_{q}\right)
\end{aligned}
$$

since $\xi \in \mathfrak{k}_{\mu}$. This shows that $\psi_{\mu}$ is well defined. It is routine to check that $\psi_{\mu}$ is smooth. In addition, $\psi_{\mu}$ is equivariant relative to the $\mathbb{R}_{+}-$ actions on $J_{c t}^{-1}\left(\mathbb{R}_{+} \mu\right) \backslash\{0\} / K_{\mu}$ and $T^{*}\left(Q / K_{\mu}\right) \backslash\{0\}$ respectively and thus it induces a smooth map on the quotients

$$
\widehat{\psi}_{\mu}:\left[\left(J_{c t}^{-1}\left(\mathbb{R}_{+} \mu\right) \backslash\{0\}\right) / K_{\mu}\right] / \mathbb{R}_{+} \rightarrow S^{*}\left(Q / K_{\mu}\right)
$$

given by

$$
\widehat{\psi}_{\mu}\left(\left[\widehat{\alpha}_{q}\right]\right)=\left[\psi_{\mu}\left(\widehat{\alpha}_{q}\right)\right]
$$

where $\left[\widehat{\alpha}_{q}\right]:=\bar{\pi}_{\mu}\left(\widehat{\alpha}_{q}\right)$, for $\bar{\pi}_{\mu}:\left(J_{c t}^{-1}\left(\mathbb{R}_{+} \mu\right) \backslash\{0\}\right) / K_{\mu} \rightarrow\left[\left(J_{c t}^{-1}\left(\mathbb{R}_{+} \mu\right) \backslash\right.\right.$ $\left.\{0\}) / K_{\mu}\right] / \mathbb{R}_{+}$is the canonical projection.

Next we show that $\widehat{\psi}_{\mu}$ is injective. If $\widehat{\psi}_{\mu}\left(\left[\widehat{\alpha}_{q}\right]\right)=\widehat{\psi}_{\mu}\left(\left[\widehat{\beta}_{q}\right]\right)$, then there exists $r \in \mathbb{R}_{+}$with $\psi_{\mu}\left(\widehat{\alpha}_{q}\right)=r \psi_{\mu}\left(\widehat{\beta}_{q}\right)$, so using $(3.11), \alpha_{q}\left(v_{q}\right)=r \beta_{q}\left(v_{q}\right)$ for every $v_{q} \in T_{q} Q$. This means that $\widehat{\alpha}_{q}=r \widehat{\beta}_{q}$ since the $K_{\mu}$ and $\mathbb{R}_{+}$ actions commute, that is, $\left[\widehat{\alpha}_{q}\right]=\left[\widehat{\beta}_{q}\right]$ showing that $\widehat{\psi}_{\mu}$ is injective. 
We need to show that $\widehat{\psi}_{\mu} \circ \bar{\lambda}_{\mu}:\left(S^{*} Q\right)_{\mu} \rightarrow S^{*}\left(Q / K_{\mu}\right)$ preserves the contact structures. Let $\Sigma: S^{*}\left(Q / K_{\mu}\right) \rightarrow T^{*}\left(Q / K_{\mu}\right) \backslash\{0\}$ be a global section and let $\Theta_{\Sigma}:=\Sigma^{*} \Theta$ be the contact form on $S^{*}\left(Q / K_{\mu}\right)$ associated to this section, where $\Theta$ is the Liouville form on $T^{*}\left(Q / K_{\mu}\right)$. The form $\Theta_{\Sigma}$ is one of the possible contact forms underlying the contact structure of the cosphere bundle $S^{*}\left(Q / K_{\mu}\right)$. Thus, to show that $\widehat{\psi}_{\mu} \circ \bar{\lambda}_{\mu}$ preserves the contact structures, it will be enough to verify that $\left(\widehat{\psi}_{\mu} \circ \bar{\lambda}_{\mu}\right)^{*} \Theta_{\Sigma}$ is proportional to $\bar{\theta}_{\sigma, \mu}$, the proportionality factor being a strictly positive function on $\left(S^{*} Q\right)_{\mu}$. Willett's contact reduction theorem at $\mu \neq 0$ states that $\bar{\theta}_{\sigma, \mu}$ is characterized by the relation $\pi_{\mu}^{*} \bar{\theta}_{\sigma, \mu}=\iota_{\mu}^{*} \theta_{\sigma}$. Thus, it suffices to show that $\left(\widehat{\psi}_{\mu} \circ \bar{\lambda}_{\mu} \circ \pi_{\mu}\right)^{*} \Theta_{\Sigma}$ is proportional to $\iota_{\mu}^{*} \theta_{\sigma}$ with a strictly positive function on $J^{-1}\left(\mathbb{R}_{+} \mu\right)$ as proportionality factor. To carry this out, we shall need a commutative diagram analogous to the one considered in Theorem 3.1.

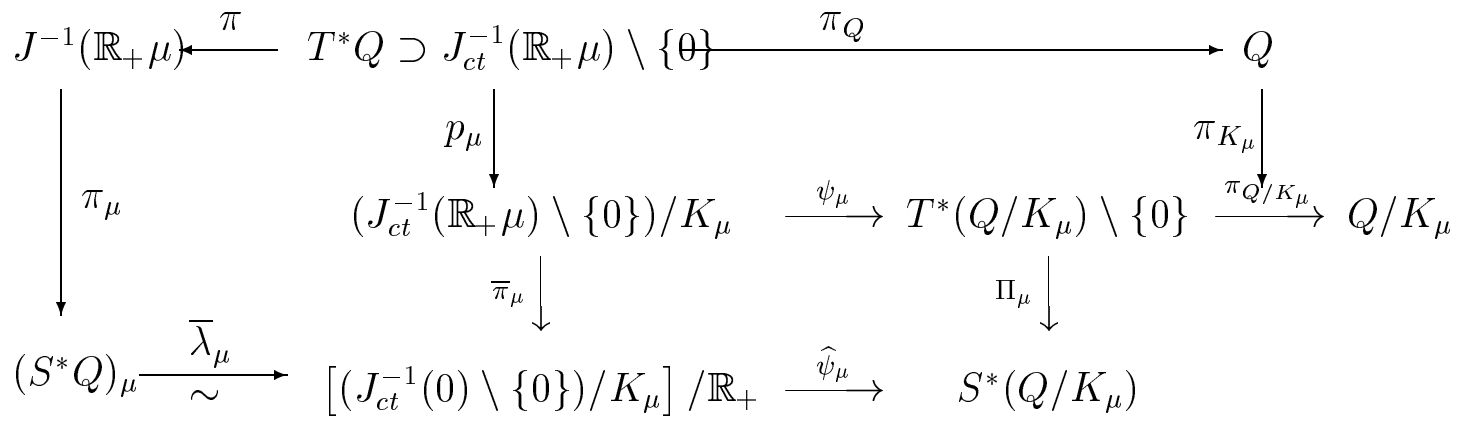

As in the proof of Theorem 3.1, we begin with the computation of $\left(\widehat{\psi}_{\mu} \circ \bar{\lambda}_{\mu} \circ \pi_{\mu} \circ \pi\right)^{*} \Theta_{\Sigma}$. Since

$$
\widehat{\psi}_{\mu} \circ \bar{\lambda}_{\mu} \circ \pi_{\mu} \circ \pi=\Pi_{\mu} \circ \psi_{\mu} \circ p_{\mu} \quad \text { and } \quad \pi_{Q / K_{\mu}} \circ \psi_{\mu} \circ p_{\mu}=\pi_{K_{\mu}} \circ \pi_{Q} \text {, }
$$

using (2.2) with base manifold $Q / K_{\mu}$, the definition (3.11) of $\psi_{\mu}$, and the global formula of the Liouville form on $T^{*}\left(Q / K_{\mu}\right)$, we get for any 
$\alpha_{q} \in J_{c t}^{-1}\left(\mathbb{R}_{+} \mu\right) \backslash\{0\}$ and any $v \in T_{\alpha_{q}}\left(J_{c t}^{-1}\left(\mathbb{R}_{+} \mu\right) \backslash\{0\}\right)$

$$
\begin{aligned}
\left(\left(\widehat{\psi}_{\mu} \circ \bar{\lambda}_{\mu} \circ \pi_{\mu} \circ \pi\right)^{*} \Theta_{\Sigma}\right)\left(\alpha_{q}\right)(v)=\left(\left(\Pi_{\mu} \circ \psi_{\mu} \circ p_{\mu}\right)^{*} \Theta_{\Sigma}\right)\left(\alpha_{q}\right)(v) \\
\quad=\left(\left(\psi_{\mu} \circ p_{\mu}\right)^{*}\left(\Pi_{\mu}^{*} \Theta_{\Sigma}\right)\right)\left(\alpha_{q}\right)(v)=\left(\left(\psi_{\mu} \circ p_{\mu}\right)^{*}\left(f_{\Sigma} \Theta\right)\right)\left(\alpha_{q}\right)(v) \\
\quad=\left(f_{\Sigma} \circ \psi_{\mu} \circ p_{\mu}\right)\left(\alpha_{q}\right)\left(\psi_{\mu} \circ p_{\mu}\right)\left(\alpha_{q}\right)\left(T_{\alpha_{q}}\left(\pi_{Q / K_{\mu}} \circ \psi_{\mu} \circ p_{\mu}\right)(v)\right) \\
\quad=\left(f_{\Sigma} \circ \psi_{\mu} \circ p_{\mu}\right)\left(\alpha_{q}\right)\left(\psi_{\mu} \circ p_{\mu}\right)\left(\alpha_{q}\right)\left(T_{\alpha_{q}}\left(\pi_{K_{\mu}} \circ \pi_{Q}\right)(v)\right) \\
\quad=\left(f_{\Sigma} \circ \psi_{0} \circ p_{\mu}\right)\left(\alpha_{q}\right) \alpha_{q}\left(T_{\alpha_{q}} \pi_{Q}(v)\right) .
\end{aligned}
$$

On the other hand, since $\iota_{\mu}: J^{-1}\left(\mathbb{R}_{+} \mu\right) \hookrightarrow S^{*} Q$ is the inclusion, from (2.2) and the definition of the Liouville form on $T^{*} Q$, we get

$$
\left(\left(\iota_{\mu} \circ \pi\right)^{*} \theta_{\sigma}\right)\left(\alpha_{q}\right)(v)=\left(f_{\sigma} \theta\right)\left(\alpha_{q}\right)(v)=f_{\sigma}\left(\alpha_{q}\right) \alpha_{q}\left(T_{\alpha_{q}} \pi_{Q}(v)\right) .
$$

The two identities (3.12) and (3.13) show that on $J_{c t}^{-1}\left(\mathbb{R}_{+} \mu\right) \backslash\{0\}$ we have the equality

$$
\pi^{*}\left(\widehat{\psi}_{\mu} \circ \bar{\lambda}_{\mu} \circ \pi_{\mu}\right)^{*} \Theta_{\Sigma}=\frac{f_{\Sigma} \circ \psi_{\mu} \circ p_{\mu}}{f_{\sigma}} \pi^{*} \iota_{\mu}^{*} \theta_{\sigma} .
$$

Formula (2.1) shows that the strictly positive proportionality factor in (3.14) drops to a strictly positive function $F_{\mu}$ on the quotient $J^{-1}\left(\mathbb{R}_{+} \mu\right)$. Since $\pi$ is a surjective submersion, (3.14) implies that $\left(\widehat{\psi}_{\mu} \circ \bar{\lambda}_{\mu} \circ \pi_{\mu}\right)^{*} \Theta_{\Sigma}=$ $F_{\mu} \iota \iota_{\mu}^{*} \theta_{\sigma}$ where the function $F_{\mu}>0$, which is the desired identity. This proves that $\widehat{\psi}_{\mu} \circ \bar{\lambda}_{\mu}$ preserves the respective contact structures.

That $\widehat{\psi}_{\mu}$ is an immersion can be proved as in the embedding version of the cotangent bundle reduction theorem (see [1], $\$ 4.3$ or [8], p. 82). Indeed, we observe that $\mu^{\prime}=\left.\mu\right|_{\mathfrak{k}_{\mu}}=0$, hence considering the action restricted to $K_{\mu}$, the corresponding momentum map $J^{\prime}$ is the restriction of $J$. We are thus in the conditions of our Theorem 3.1 and obtain a contact-diffeomorphism between $J^{\prime-1}\left(\mathbb{R}_{+} \mu^{\prime}\right) / K_{\mu}=J^{\prime-1}(0) / K_{\mu}$ and $S^{*}\left(Q / K_{\mu}\right)$. Composing this with the natural inclusion of $J^{-1}\left(\mathbb{R}_{+} \mu\right) / K_{\mu}$ in $J^{\prime-1}\left(\mathbb{R}_{+} \mu^{\prime}\right) / K_{\mu}$, we arrive at the desired contact embedding.

This ends the proof of the theorem.

Example 3.4. We look again at Albert's example discussed above. We have $Q=\mathbb{T}^{n}, G=\mathbb{T}^{n}$ acting naturally on itself. Take $\mu \in\left(\mathbb{R}^{n}\right)^{*}$ to be the projection on the last factor: $\mu\left(x_{1}, \ldots, x_{n}\right)=x_{n}$. Then ker $\mu=$ $\mathbb{R}^{n-1}, K_{\mu}=\mathbb{T}^{n-1}$ and $J^{-1}\left(\mathbb{R}_{+} \mu\right) \cong \mathbb{T}^{n}$. Hence $\left(S^{*} Q\right)_{\mu}=\mathbb{T}^{n} / \mathbb{T}^{n-1} \cong S^{1}$ and $Q / K_{\mu} \cong S^{1}$. Our theorem yields $S^{1} \hookrightarrow S^{*}\left(S^{1}\right)$, the inclusion being the zero section in $T^{*} S^{1}$ followed by the canonical projection. 
Example 3.5. We let $Q=\mathbb{R}^{3(n+1)}$ and $\varphi$ be the natural action of $G=\left(\mathbb{R}^{3},+\right)$ on $Q$ by translations. The lifted action to the cotangent bundle is again by translations: $\left(\mathbf{x},\left(\mathbf{q}_{i}, \mathbf{p}^{i}\right)\right) \mapsto\left(\mathbf{x}+\mathbf{q}_{i}, \mathbf{p}^{i}\right), i=0, \ldots, n$. The symplectic momentum map (the linear momentum, see e.g. [8]) has values in $\mathbb{R}^{3}$ (which is identified with its dual $\left(\mathbb{R}^{3}\right)^{*}$ by the usual dot product) and is given by:

$$
J_{c t}\left(\mathbf{q}_{i}, \mathbf{p}^{i}\right)=\sum_{j=0}^{n} \mathbf{p}^{j} .
$$

Fix now $\mathbf{v} \in \mathbb{R}^{3} \backslash\{0\}$ and define $\mu: \mathbb{R}^{3} \rightarrow \mathbb{R}$ by $\mu(\xi)=\mathbf{v} \cdot \xi$. Then we have:

$$
J_{c t}^{-1}\left(\mathbb{R}_{+} \mu\right)=\left\{\left(\mathbf{q}_{i}, \mathbf{p}^{i}\right) \mid \sum_{i=0}^{n} \mathbf{p}^{i} \in \mathbb{R}_{+} \mathbf{v}\right\} .
$$

As $G$ is Abelian, we have $\mathfrak{k}_{\mu}=\operatorname{ker} \mu=\mathbf{v}^{\perp} \cong \mathbb{R}^{2}$. Hence $K_{\mu} \cong \mathbb{R}^{2}$. Define the map $f: \mathbb{R}^{3(n+1)} \rightarrow \mathbb{R}^{3 n+1}$ by

$$
f\left(\mathbf{q}_{0}, \ldots, \mathbf{q}_{n}\right):=\left(\mathbf{q}_{1}-\mathbf{q}_{0}, \ldots, \mathbf{q}_{n}-\mathbf{q}_{n-1}, \mathbf{q}_{0} \cdot \mathbf{v} /\|\mathbf{v}\|^{2}\right) .
$$

Clearly $f$ is smooth, surjective, invariant under the $K_{\mu}$-action, and $f\left(\mathbf{q}_{0}, \ldots, \mathbf{q}_{n}\right)=f\left(\mathbf{q}_{0}^{\prime}, \ldots, \mathbf{q}_{n}^{\prime}\right)$ if and only if $\mathbf{q}_{i}^{\prime}=\mathbf{q}_{i}+\mathbf{x}$, for all $i=$ $0, \ldots, n$, where $\mathbf{x} \in \mathbf{v}^{\perp}$. In addition, the kernel of the derivative of $f$ at every point equals the tangent space the $K_{\mu}$-orbit. Hence $f$ induces a diffeomeorphism $Q / K_{\mu}=\mathbb{R}^{3(n+1)} / \mathbb{R}^{2} \cong \mathbb{R}^{3 n+1}$. We thus have:

$$
S^{*}\left(Q / K_{\mu}\right)=S^{*} \mathbb{R}^{3 n+1} \cong \mathbb{R}^{3 n+1} \times S^{3 n} .
$$

On the other hand,

$$
J_{c t}^{-1}\left(\mathbb{R}_{+} \mu\right) / K_{\mu} \cong \mathbb{R}^{3 n+1} \times\left\{\left(\mathbf{p}^{0}, \ldots, \mathbf{p}^{n}\right) \mid \sum_{i=0}^{n} \mathbf{p}^{i} \in \mathbb{R}_{+} \mathbf{v}\right\}
$$

since the $K_{\mu}$-action does not affect $\mathbf{p}^{0}, \ldots, \mathbf{p}^{n}$. Applying Theorem 3.2 we have :

$$
\left(S^{*} Q\right)_{\mu} \cong \mathbb{R}^{3 n+1} \times\left[S^{3 n+2} \cap\left\{\left(\mathbf{p}^{0}, \ldots, \mathbf{p}^{n}\right) \mid \sum_{i=0}^{n} \mathbf{p}^{i} \in \mathbb{R}_{+} \mathbf{v}\right\}\right]
$$

So Theorem 3.2 asserts the existence of a contact structure on the above manifold, induced from that of $\mathbb{R}^{3 n+1} \times S^{3 n}$. Note that it is not obvious how to construct directly a contact structure on

$$
\mathbb{R}^{3 n+1} \times\left[S^{3 n+2} \cap\left\{\left(\mathbf{p}^{0}, \ldots, \mathbf{p}^{n}\right) \mid \sum_{i=0}^{n} \mathbf{p}^{i} \in \mathbb{R}_{+} \mathbf{v}\right\}\right] .
$$


Remark 3.2. Observe that $\widehat{\psi}_{\mu}$ may no longer be surjective (as the corresponding map of the symplectic case). In fact, since $\widehat{\psi}_{\mu}$ maps fibers of $J^{-1}\left(\mathbb{R}_{+} \mu\right) / K_{\mu}$ in fibers of $S^{*}\left(Q / K_{\mu}\right)$, if it were surjective it would be so on each fiber, but a simple count of dimensions proves this is impossible. On the other hand, conditions like $G=K_{\mu}$ or $\mathfrak{g}=\mathfrak{g}_{\mu}$, which ensure surjectivity in the symplectic case, here lead to $\mu=0$ (because of the condition ker $\mu+\mathfrak{g}_{\mu}=\mathfrak{g}$ ).

Remark 3.3. There is a significant difference between the reduced spaces for $\mu \neq 0$ in the cotangent bundle reduction theorem and for the cosphere bundle. The symplectic quotient is symplectically embedded (only in the particular case of $G$ Abelian or $G=G_{\mu}$ one obtains a diffeomorphism) in $T^{*}\left(Q / G_{\mu}\right)$ endowed with a perturbed symplectic form (the canonical one minus a magnetic term), while the contact quotient is always contactly embedded in $S^{*}\left(Q / K_{\mu}\right)$ with a non perturbed contact form. Thus, in contact geometry, the cases $\mu \neq 0$ and $\mu=0$ are similar and the explanation is Willett's choice of the kernel group of $\mu$ instead of the coadjoint isotropy group of $\mu$. Explicitly, it is the Lie algebra of this kernel group that assures the existence of a well-defined map preserving the contact structure exactly as in the $\mu=0$ case.

Remark 3.4. One may relax the assumptions on the action of $G$ (and $K_{\mu}$ ) by allowing fixed points and working in the category of orbifolds. 
Acknowledgment. L.O. is a member of EDGE, partially supported by the European Contract "Human Potential Programme Research Training Network" HPRN-CT-2000-00101 and by the Swiss NSF through the SCOPES Program during a one month visit at the EPFL. T.S.R. was partially supported by the European Commission and the Swiss Federal Government through funding for the Research Training Network Mechanics and Symmetry in Europe (MASIE) as well as the Swiss National Science Foundation. We would like to thank P. Birtea, I. Marshall, J.-P. Ortega, T. Schmah for many useful conversations during the elaboration of this work. 


\section{Bibliography}

[1] R. Abraham, J. Marsden, Foundations of Mechanics, second edition, New York, Benjamin/Cummings, 1978.

[2] C. Albert, Le théorème de réduction de Marsden-Weinstein en géométrie cosymplectique et de contact, J. Geom. Physics, 6 (1989), 627-649.

[3] V.I. Arnold, Mathematical Methods of Classical Mechanics, Springer Verlag, 1984.

[4] H. Geiges, Constructions of contact manifolds, Math. Proc. Cambridge Philos. Soc., 121 (1997), 455-464.

[5] E. Lerman, Contact cuts, Israel. J. Math. 124 (2001), 77-92.

[6] J.E. Marsden, Lectures on Mechanics, London Math. Soc. Lecture Notes Series 174, Cambridge Univ. Press., 1992.

[7] J.E. Marsden, T.S. Ratiu, Introduction to Mechanics and Symmetry, Springer Texts in Appl. Math. 17, Second edition, 1999.

[8] J.E. Marsden, T.S. Ratiu, Introduction to Mechanics and Symmetry II. Reduction theory, manuscript.

[9] T. Ratiu, R. Schmid, The differentiable structure of three remarkable diffeomorphism groups, Math. Z., 177 (1981), 81-100.

[10] W.J. Satzer, Canonical reduction of mechanical systems invariant under Abelian group actions with an application to celestial mechanics, Indiana Univ. Math. Journ., 26 (1977), 951-976.

[11] C. Willett, Contact reduction, Trans. Amer. Math. Soc., 354 (2002), no. 10, 4245-4260. 
714 OANA DRĂGULETE, LIVIU ORNEA, TUDOR S. RATIU

Department of Mathematics, University "Politehnica" of Bucharest, Bucharest, Romania AND

Institut Bernoulli, EPFL, CH-1015

Lausanne, Switzerland

E-mail address: oana.dragulete@epfl.ch

University of Bucharest, Faculty of Mathematics

14 Academiei str., 70109 Bucharest, Romania

E-mail address: lornea@imar.ro

Institut Bernoulli, EPFL, CH-1015 Lausanne, Switzerland

E-mail address: Tudor.Ratiu@epfl.ch

This version: May 17, 2003. 